
situ measurements of unexpectedly large $\mathrm{HNO}_{3}$-containing particles in the Arctic vortex

\author{
S. Molleker et al.
}

Correspondence to: S. Borrmann (stephan.borrmann@mpic.de) 


\section{Meteorology and Flight Tracks}

The supplementary Figs. S1 and $\mathbf{S 2}$ show the temperature distribution on the $50 \mathrm{hPa}$ levels, which is closer to the flight altitude of the M-55 Geophysica. The temperature maps at $30 \mathrm{hPa}$ from Fig. 01 and Fig. 03 is more related to the higher altitudes, where large PSC-particles must have been nucleated at first. Growth followed by sedimentation transported them downwards prior to their detection at the flight levels. The contours of the $30 \mathrm{hPa}$ and $50 \mathrm{hPa}$ maps are similar; only the general temperature levels at $50 \mathrm{hPa}$ are approximately 3-5 degrees higher. In addition Figure S3 provides more detailed information on the flight paths than discernible from Figs. 01 and 03.

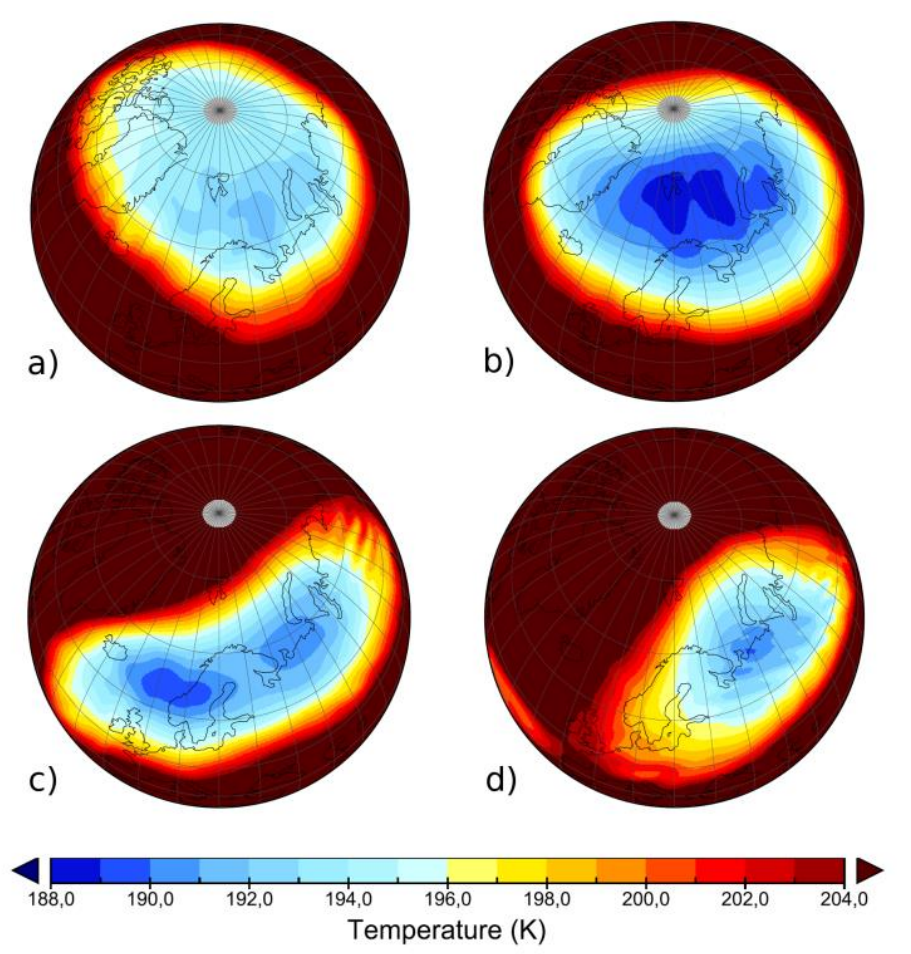

Figure S1: ERA Interim data of temperatures on the $\mathbf{5 0} \mathrm{hPa}$ level during RECONCILE times in January 2010: a) 10 January 00:00 UTC, b) 17 January 12:00 UTC - corresponding to the first PSC flight, c) 25 January 12:00 UTC i.e. the last flight with PSC encounter, d) 28 January 18:00 UTC. The darkest blue color in the plot highlights areas where temperatures reached saturation $\left(T_{\text {ICE }}\right)$ with respect to ice ( $\left.T \leq 188 \mathrm{~K}\right)$. 


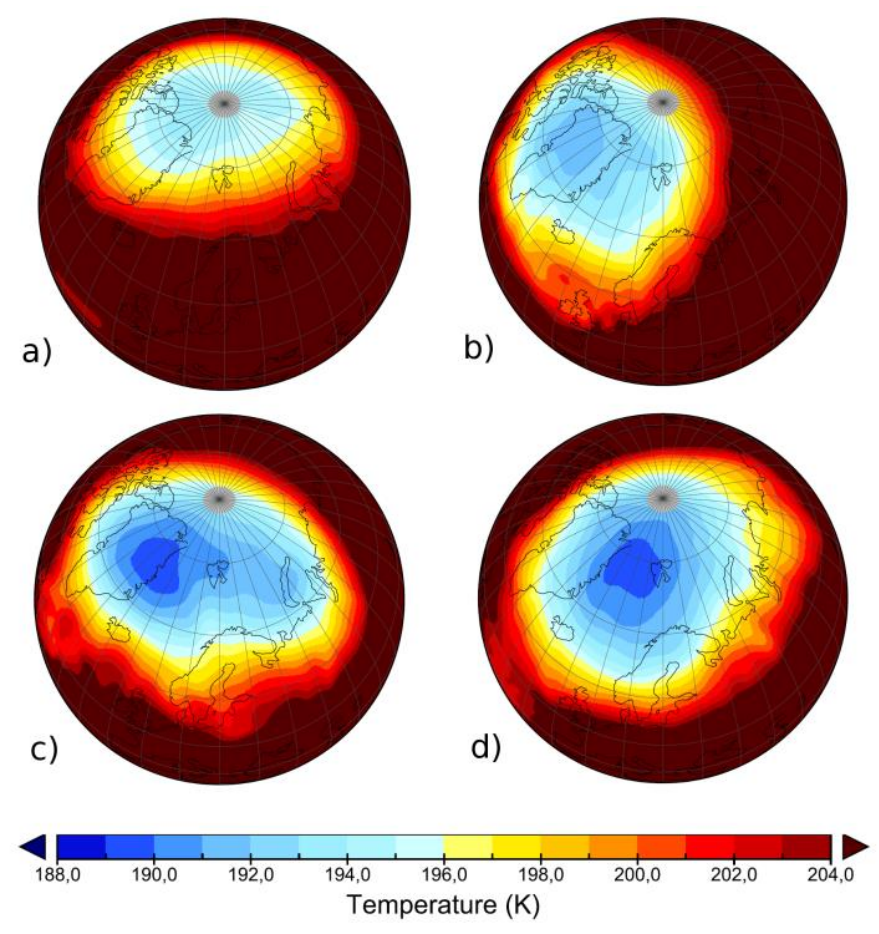

Figure S2: ERA Interim data of temperatures on the $50 \mathrm{hPa}$ level during the ESSenCe campaign for the following times in December 2011: a) 4 December 00:00 UTC, b) 8 December 18:00 UTC, c) 11 December 12:00 UTC - corresponding to the first flight, d) 16 December 18:00 UTC - close to the second flight. The polar vortex established during December 2011.

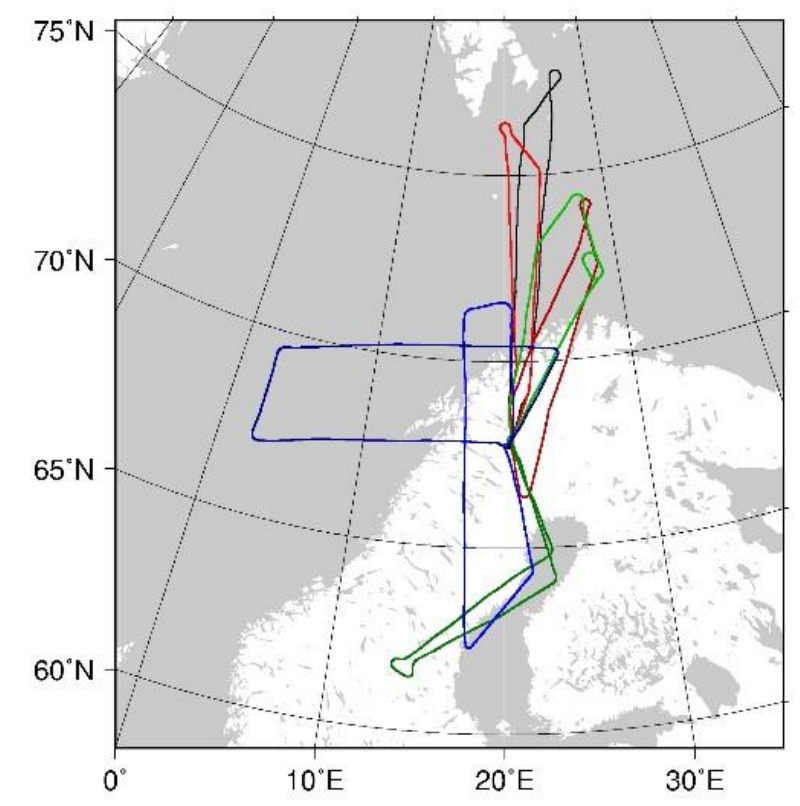

Figure S3: The flight tracks of five PSC-Flights from the RECONCILE-Campaign performed in January 2010 are shown together with two additional flights performed in December 2011 (ESSenCe-Campaign). All flights were performed out of Kiruna, Sweden. RECONCILE-Flights numbers 1 to 5 are marked: black (17 January 2010), red (20 January 2010), dark red (22 January 2010), green (24 January 2010), dark green (25 January 2010). ESSenCe flights: blue (11 December 2011) and dark blue (16 December 2011). 


\section{Note on the chemical analyses of aerosol samples collected outside and inside PSCs}

During the RECONCILE and ESSenCe flights within the polar vortex also impactor sampling of particles in the size range $100 \mathrm{~nm}-5 \mu \mathrm{m}$ was conducted. Within 11 RECONCILE flight-samples a variety of large non-volatile particles (with size diameters from $500 \mathrm{~nm}$ to $5 \mu \mathrm{m}$ ) could be collected for a posteriori analyses in the laboratory. Analyses are provided of the size, chemical composition and the morphology of individual particles within these samples by scanning electron microscopy by Ebert et al., 2015. Briefly, the 759 analyzed non-volatile particles were found to be around $30 \%$ silicates, $20 \%$ iron rich and $6 \%$ carbon/silicate mixtures. Many of these particles will be of extraterrestrial origin. Around $10 \%$ of the particles were Ca-rich, which also was found by Corte et al., 2013, and $30 \%$ of the particles show a composition which is similar to certain anthropogenic metal alloys (including $\mathrm{Al}$, $\mathrm{Cr}, \mathrm{Mn}, \mathrm{Fe}, \mathrm{Ni}, \mathrm{Cu}, \mathrm{Zn}$ ). In the NASA cosmic dust catalog (Warren et al., 2011) such "alloy-like" stratospheric particles are partly classified as of cosmic and partly as of terrestrial origin.

In contrast to these findings for PSC free conditions large non-volatile particles were almost completely absent in all RECONCILE and ESSenCe samples, which were taken during PSC events. Inside the PSC only a little number (116) of small $(<500 \mathrm{~nm}$ ) non-volatile particles could be sampled. Besides around $35 \% \mathrm{Mg}$-rich silicates and $25 \%$ Fe-rich particles, most likely of meteoric origin, also $25 \%$ aluminum oxide spheres possibly from rocket launches were detected, as well as $15 \%$ lead-rich particles from an unknown source. For future research flights inside PSCs it seems important to deploy a counterflow virtual impactor unit for separate sampling of PSC and interstitial particles in order to assess the chemical composition of the cloud nuclei.

\section{Measurement Techniques: In-situ optical particle counters and data availability}

Table S1 provides information on the aerosol, cloud particle, and NOy instrumentation implemented on the M-55 Geophysica.

\begin{tabular}{|c|c|c|c|c|c|c|}
\hline $\begin{array}{l}\text { Instrument: } \\
\text { Detection limit: }\end{array}$ & $\begin{array}{c}\text { COPAS } \\
\mathrm{Dp}>15 \mathrm{~nm}\end{array}$ & $\begin{array}{c}\text { FSSP-300 } \\
\text { Dp }>0,46 \mu \mathrm{m}\end{array}$ & $\begin{array}{c}\text { FSSP-100 } \\
\text { Dp }>1,05 \mu \mathrm{m}\end{array}$ & $\begin{array}{c}\mathrm{CDP} \\
\mathrm{Dp}>5 \mu \mathrm{m}\end{array}$ & $\begin{array}{c}\text { CIPgs } \\
\text { Dp }>10-15 \mu \mathrm{m}\end{array}$ & $\begin{array}{l}\text { SIOUX (NOy) } \\
\text { Dp }>5-12 \mu \mathrm{m}\end{array}$ \\
\hline \multicolumn{7}{|c|}{ RECONCILE flights } \\
\hline 17 Jan 2010 & $\mathrm{x}$ & $\mathrm{x}$ & $\mathrm{x}$ & - & - & - \\
\hline 20 Jan 2010 & $\mathrm{x}$ & $\mathrm{x}$ & - & - & $\mathrm{x}$ & - \\
\hline 22 Jan 2010 & $\mathrm{x}$ & - & $\mathrm{x}$ & - & $\mathrm{x}$ & - \\
\hline 24 Jan 2010 & $\mathrm{x}$ & - & $\mathrm{x}$ & - & $\mathrm{x}$ & $\mathrm{x}$ \\
\hline 25 Jan 2010 & $\mathrm{x}$ & - & $\mathrm{x}$ & $\mathrm{x}$ & $\mathrm{x}$ & $\mathrm{x}$ \\
\hline \multicolumn{7}{|l|}{ ESSenCe flights } \\
\hline 11 Dec 2011 & $\mathrm{x}$ & - & $\mathrm{x}$ & $\mathrm{x}$ & - & - \\
\hline 16 Dec 2011 & $\mathrm{x}$ & - & $\mathrm{x}$ & $\mathrm{x}$ & - & - \\
\hline
\end{tabular}

Table S1: Data availability of six single-particle detecting instruments from two Arctic field campaigns together with their detection limits in terms of particle size diameter $D p$. COPAS is a COndensation Particle Counting System, FSSP-100/300 and CDP are Forward Scattering Spectrometer Probes, CIPgs is an optical array probe (Cloud Imaging Probe, grey scale) and SIOUX is an instrument measuring the gas phase NOy with a forward (and a backward) facing inlet resolving single sufficiently large NOy containing particles.

Data quality of the FSSP-300: Since the FSSP-300 on the M-55 Geophysica has undergone several modifications (e.g. of hardware, control software and alignments; not the optics) between its first deployment in the Arctic lower stratosphere in 1996 (Borrmann et al., 2000, and 2010), specific time 
periods of both data sets were selected. These cover periods with comparable background aerosols inside and outside of the polar vortices, which represent particles with sizes at the FSSP-300's lower detection limit. For these smallest particles the instrument is most susceptible with respect to alignment errors and electronic noise problems. The corresponding comparison of size distributions is shown in Fig. S4. With the given uncertainty in counting statistics the background aerosol size distributions agree quite well and the conclusion is that the instrument's main characteristics have not changed throughout all the implemented modifications.

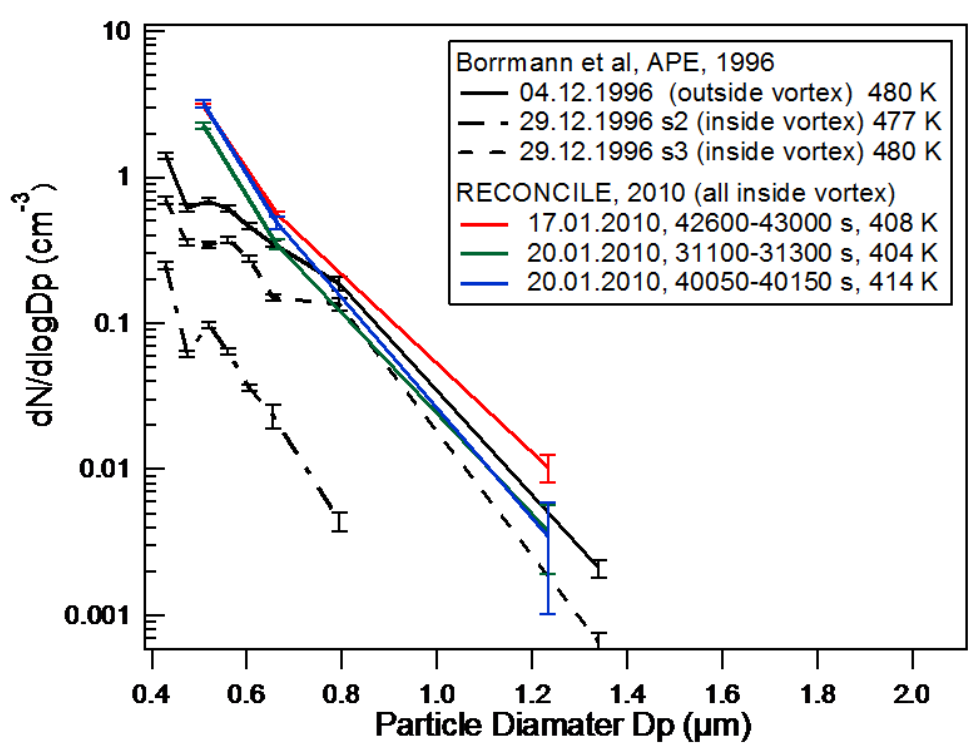

Figure S4: FSSP-300 size distributions of background aerosol from different Northern hemispheric polar winter vortices (the altitude levels are indicated in the legend in terms of potential temperature in Kelvin). Here only measurements outside of PSCs from 2010 are juxtaposed with measurements of the same instrument during the 1996 APE-Campaign (Airborne Polar Experiment; Borrmann et al. 2000). After 2000 the instrument was upgraded with a new digital signal processing module (SPP-300 from DMT, Boulder, Co, USA; among other modifications). Here this data is merely considered for instrument inter-comparison and compatibility purposes, as the measurements show a consistent picture. Note however, the size distribution denoted as "s2" is deep inside the vortex where 1997 record low particle number densities were found (Borrmann et al., 2000). Disregarding "s2" the background number concentrations are very similar considering also the different polar vortices.

\section{Measurement Techniques: Details on Lidar and Backscattersonde Instruments}

The two optical instruments MAL and MAS are well established, described in the peer reviewed literature (Cairo et al., 2011, Matthey, 2000), and have been deployed on the M-55 Geophysica since its first campaign in 1996. Since the measurements during RECONCILE and inside PSCs are a specialized application, the instrument performances and characteristics are summarized here.

\subsection{Multiwavelength Aerosol Scatterometer (MAS)}

The backscattersonde emits polarized light at $532 \mathrm{~nm}$ and $1064 \mathrm{~nm}$ and collects the backscattered photons from the atmosphere in the proximity of the instrument. The light detection starts three meters from the aircraft with decreasing efficiency, which approaches 0 at $100 \mathrm{~m}$ distance. By means 
of the backscattered light ensembles of aerosol and cloud particles with diameters greater than a few tenths of $\mu \mathrm{m}$ can be detected. Polarization resolved observation then also allows to discriminate the shape -hence the thermodynamic phase- of the scatterers. The instrument is basically a polarization diversity Rayleigh lidar measuring in situ the parameters accessible to lidars (i.e. the scattering ratio $S R$ and the depolarization ratio $D R$ ) defined as:

(S1) $\quad S R=\frac{\beta_{m o l}+\beta_{a e r}}{\beta_{m o l}}$

$$
D R=\frac{\beta_{S, \mathrm{~mol}}+\beta_{S, a e r}}{\beta_{P, \mathrm{~mol}}+\beta_{P, a e r}}
$$

Here $\beta_{\mathrm{x}, \mathrm{n}}$ are the volume backscatter coefficients for molecules and aerosols ( $n$ being either $\mathrm{mol}$, or aer, and $X$ either $P$ or $S$ ), expressed in $\mathrm{m}^{-1} \mathrm{sr}^{-1}$, as defined in Collis and Russell (1976):

$$
\beta_{m o l}=N * \frac{d \sigma_{m o l}}{d \Omega}
$$

$$
\beta_{a e r}=\int_{0}^{\infty} n(r) \frac{d \sigma_{a e r}(r)}{d \Omega} d r
$$

In these equations $N$ denotes the molecular number concentration, $n(r)$ is the particle size distribution (i.e. the number of particles with size radii $r$ between $r$ and $r+d r$ in a unit volume), and $\frac{d \sigma_{\text {mol,aer }}}{d \Omega}$ the backscattering cross section for molecules and aerosol. The subscripts $P$ and $S$ in Eq. (S2) designate their polarized and depolarized contributions.

In the configuration adopted during RECONCILE MAS uses a Nd-YAG laser, which is actively Qswitched at $0.3 \mathrm{~s}^{-1}$ pulse repetition rate. The energy per pulse on its SHG emission (532 nm) is $5 \mathrm{~mJ}$. The light polarization, specified 100:1 by the manufacturer, is further cleaned by a cube polarizer (500:1 extinction ratio) that filters the light before its emission into the atmosphere. The backscattered light is divided into the two polarization planes by a similar polarizing cube and then further cleaned by a second cube placed in front of the photomultipliers. The parallelism between the polarization plane of the laser and the axes of the polarizers has been designed, implemented, and optically controlled to stay below $0.25^{\circ}$. Deviations are the main source of inaccuracy in the depolarization measurements. The induced cross-talk between the channels is estimated to stay below $0.5 \%$. At its maximum value, and given the depolarization calibration procedure detailed below, this systematic effect can in principle depress the aerosol depolarization by as much as $20 \%$ in the range of values presented in Fig. 010 (as shown for example by the simulations in Cairo et al., 1999). However, for RECONCILE the depolarizations encountered in cirrus clouds have been checked, and their values stayed within the range reported in the literature. If there had been a cross talk effect, it did not significantly influence the cloud classifications or the main message inherent in Fig. 010. According to Fig. 010 only weakly depolarizing clouds with a progressive enhancement due to the presence of aspherical scatterers were detected (as seen in the observations from the flights of 17 January and of 20 to 25 January 2010). 
For the calibration of the scattering ratio $S R$ and the volume depolarization ratio $D R$ suitable constants $C R$ and $C D$ at a time $t_{0}$ are derived from flight segments where the airplane crossed air supposedly free of clouds (the "calibration range"). (This was checked by inspecting the data from the aerosol instruments on the M-55 Geophysica.) The implicit relations for $C R$ and $C D$ are:

(S5) $\quad P_{P}\left(t_{0}\right)=C R \frac{p\left(t_{0}\right)}{T\left(t_{0}\right)}$

(S6) $\frac{P_{S}\left(t_{0}\right)}{P_{P}\left(t_{0}\right)} C D=D R_{\text {mol }}$,

where $P_{P}(t)$ and $P_{S}(t)$ designate the raw backscattered signals observed during the flight, and $p$ and $T$ stand for the pressures and temperatures from other sensors on board. A volume depolarization of $D R_{\text {mol }}=0.014$ has to be expected for a molecular atmosphere, given the $10 \mathrm{~nm}$ FWHM bandwidth of the MAS interferential filter (Young, 1980; Behrendt and Nakamura, 2002). Considering the broad FWHM all measurements related to the assessment of $C R$ and $C D$ were performed in darkness. The parallel backscatter and depolarization ratios can be calculated from:

$$
S R_{P}(t)=\frac{P_{P}(t)}{C R \frac{p(t)}{T(t)}}
$$

$$
D R(t)=\frac{P_{S}(t)}{P_{P}(t)} C D
$$

and the total backscatter ratio as:

(S9) $S R(t)=\frac{S R_{P}(t)[1+D R(t)]}{1+D R_{\text {mol }}}$.

This "zero-degree calibration procedure" (Freudenthaler et al., 2009), although commonly used, might lead to inaccuracies in the depolarization values, when small amounts of aerosol are present in the calibration range. Thus, the results are checked in the laboratory after the field deployment with a method outlined in Snels et al. (2009), which is similar to the one by Freudenthaler et al. (2009).

\subsection{Miniature Airborne Lidar (MAL)}

The two MAL lidars are applied on the M-55 Geophysica for the detection of polar stratospheric clouds similar to the ground based lidar by Achtert and Tesche, 2014. The procedures to determine the backscatter and depolarization ratios for MAL are described in Mitev et al. (2002) and briefly summarized here for the RECONCILE data set.

The upward directed MAL1 and the downward pointing MAL2 instruments are identical elastic backscatter-depolarization lidars operating at $532 \mathrm{~nm}$ wavelength. The established method to determine the total scattering ratio is based on a comparison with the pure molecular backscatter of 
the air. The equation for the received power for MAL1 and MAL2 operating at short range from the high altitude aircraft M-55 Geophysica can be simplified by assuming that the atmospheric attenuation within the probed volume is negligible. The value for the background and dark noise corrected signal $S_{C}(z)$ from a distance $z$ can be obtained (after normalizing to the laser energy transmitted during the integration time, and to the range resolution, as well as after further correcting for the range to the probed volume) by the following equation:

$$
S_{C}(z)=\frac{N(z)-N_{B}}{E_{L} \Delta z} \cdot z^{2}=C \cdot \beta(z)=C \cdot\left[\beta_{m o l}(z)+\beta_{a e r}(z)\right]=C \cdot \beta_{m o l}(z) \cdot S R(z)
$$

Here $N(z)$ is the detected signal from bin $\Delta z$ at distance $z$ in photon counts and $N_{B}$ denotes the number of photon counts due to the optical background and dark noise of the photomultiplier. The parameters $\beta_{m o l}(z)$ and $\beta_{a e r}(z)$ are the same variables as defined in Eq. (S3) and Eq. (S4), i.e. the molecular and aerosol backscatter coefficients defined as average over a distance bin width $\Delta z$ centered at distance $z$ from the lidar. The Eq. (S10) is valid for a single measurement at any time $t$ during the flight. The constant $C$ is determined at range $z_{0}$ (and time $t_{0}$ ), which is the calibration range where the lidar(s) probe cloud free atmosphere. At these ranges and times the value for $C$ is determined from the temperature and pressure profiles in the atmosphere corrected with respect to the measured temperatures and pressures at the aircraft's flight level. Thus:

$$
C=\frac{S_{C}\left(z_{0}, t_{0}\right)}{\beta_{m o l}\left(z_{0}, t_{0}\right)}
$$

After determination of the calibration constant $C, S R(z)$ is determined from Eq. (S10).

The laser light is linearly polarized with a ratio of approximately 1:100. It passes a polarizer and is finally is transmitted as linearly polarized with a ratio of about 1:500. The interference filter in the receiver chain has $F W H M=0.12 \mathrm{~nm}$. Unlike as it is in MAS, for such a filter bandwidth only the Cabannes line passes (Behrendt and Nakamura, 2002), i.e., no pure rotational Raman spectrum is transmitted. The volume depolarization ratio of the molecular backscatter through this filter is $0.368 \%$. Here the temperature dependence in the volume depolarization ratio measurements does not need to be taken into account (Behrendt and Nakamura, 2002).

With $\beta_{P, n}(z)$ and $\beta_{S, n}(z)$ (n being either mol, or aer) denoting the atmospheric backscatter at a polarization plane respectively parallel and perpendicular to the one of the transmitted laser light, the volume depolarization ratio $D R(z)$ is then determined for MAL similar to Eq. (S8) by:

$$
D R(z)=\frac{\beta_{S, m o l}(z)+\beta_{S, a e r}(z)}{\beta_{P, m o l}(z)+\beta_{P, a e r}(z)}=\frac{S_{c, S}(z)}{R \cdot S_{c, P}(z)}
$$

As in Eq. (S2) the variables $D R(z) ; \beta_{S, \text { mol }}(z), \beta_{P, \text { mol }}(z), \beta_{S, \text { aer }}(z)$, and $\beta_{P, \text { aer }}(z)$ represent the volume depolarisation ratio and the molecular, and aerosol backscatter coefficients at polarizations " $S$ " and " $P$ ". For a distance $z$ from the lidar the signal is averaged over a bin width $\Delta z$ centred on $z$. The parameters $S_{c, S}(z)$ and $S_{c, P}(z)$ are the signals after the corrections for range and transmitted power. The calibration constant $R$ is the ratio of the efficiency constants of the lidar for the perpendicular and parallel signal channels of the receiver and detection chains. It is determined by a 
calibration where the polarization of the transmitted light is set to 45 degrees with respect to the polarization plane used during the aircraft measurements.

The aerosol depolarization can be derived from the volume depolarization by

$$
D R_{\text {aer }}(z)=\frac{\beta_{S, a e r}(z)}{\beta_{P, a e r}(z)}
$$

as described in Cairo et al. (1999). This aerosol depolarization $D R_{a e r}$ from Eq. (S13) and the total backscatter ratios $S R$ (based on Eq. (S9)) are reported in the text and Fig. 010, while Fig. 07 shows the measured volume depolarization ratio.

Additional verification of the measurements of MAL1 and MAL2 was performed by comparison with the measurements of the CALIPSO lidar. The measurements of MAL1 and MAL2 on the M-55 Geophysica and of the CALIPSO lidar (also at 532nm; Pitts et al., 2009) have been co-located during the Geophysica flight on 20 January 2010. The overlap between the aircraft flight path and the CALIPSO track lasted approximately 12 minutes. The comparison was performed for the backscatter at parallel and perpendicular polarizations, instead of backscatter and depolarization ratios (Mitev et al., 2012). Figures S5 to S8 show the results of the comparison between the profiles of the total backscatter coefficient measured by the two MALs (solid red line, downward looking MAL; solid cyan line, upward oriented MAL) and the CALIPSO lidar (solid blue line). The dashed (blue and red) lines represent the one-sigma variability of the CALIPSO profile averaged over the aircraft measurement time window. At the altitudes of PSC occurrence the agreement is satisfactory as the differences are well within one standard deviation. The comparison at cirrus altitudes is also good. Here the colocation between the respective probing tracks was not exact and the small deviations may be due to the different sampling points. Please note that on Figures $\mathrm{S} 5$ to $\mathrm{S} 8$ "beta total" stands for $\beta_{\text {mol }}(z)+$ $\beta_{\text {aer }}(z)$ or $\beta_{S, \text { mol }}(z)+\beta_{S, \text { aer }}(z)+\beta_{P, \text { mol }}(z)+\beta_{P, a e r}(z)$; while the term "beta depolarisation" refers to $\beta_{S, m o l}(z)+\beta_{S, a e r}(z)$. 


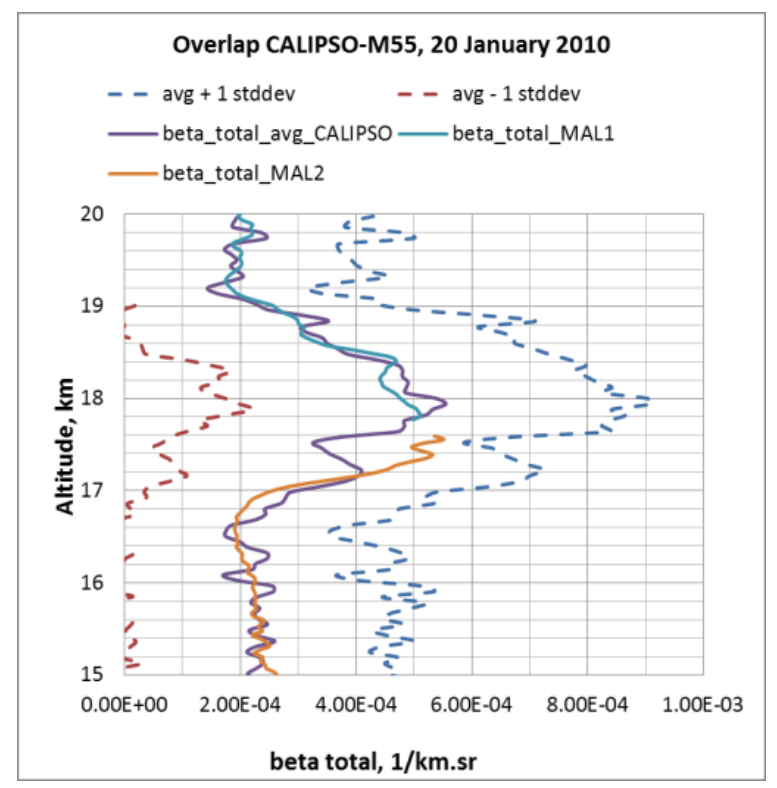

Figure S5: Comparison at PSC altitudes: Profiles of the total backscatter coefficient derived from the measurements of the CALIPSO lidar, MAL1 and MAL2, and averaged from $35800 \mathrm{~s}$ to $36600 \mathrm{~s}$ UTC for the flight on 20 January 2010. The dashed lines indicate the one-sigma variability in the CALIPSO lidar.

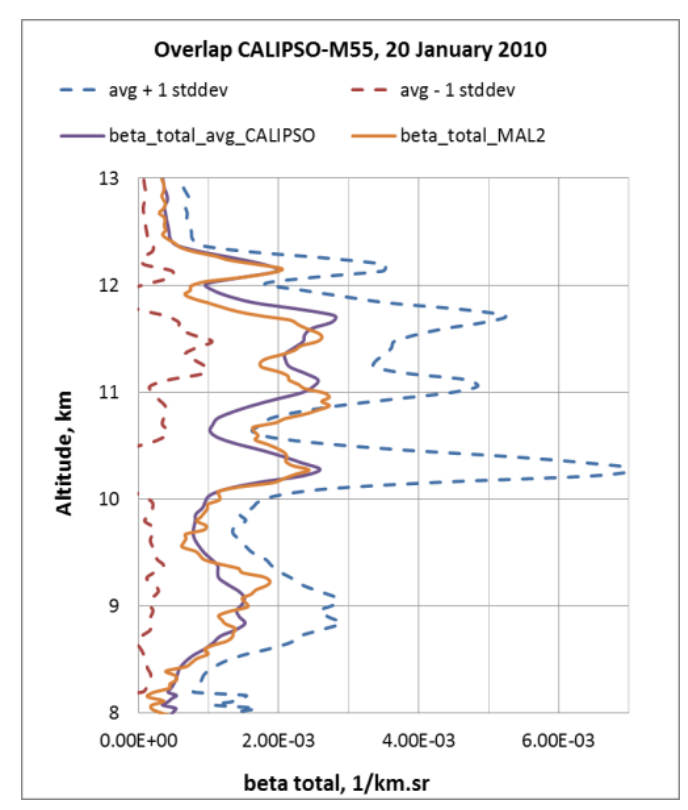

Figure S6: Comparison at cirrus altitudes: Profiles of the total backscatter coefficient derived from the measurements of the CALIPSO lidar, MAL1 and MAL2 as in Fig. S5. Averaging period from $35800 \mathrm{~s}$ to $36600 \mathrm{~s}$ UTC of the flight from 20 January 2010. 


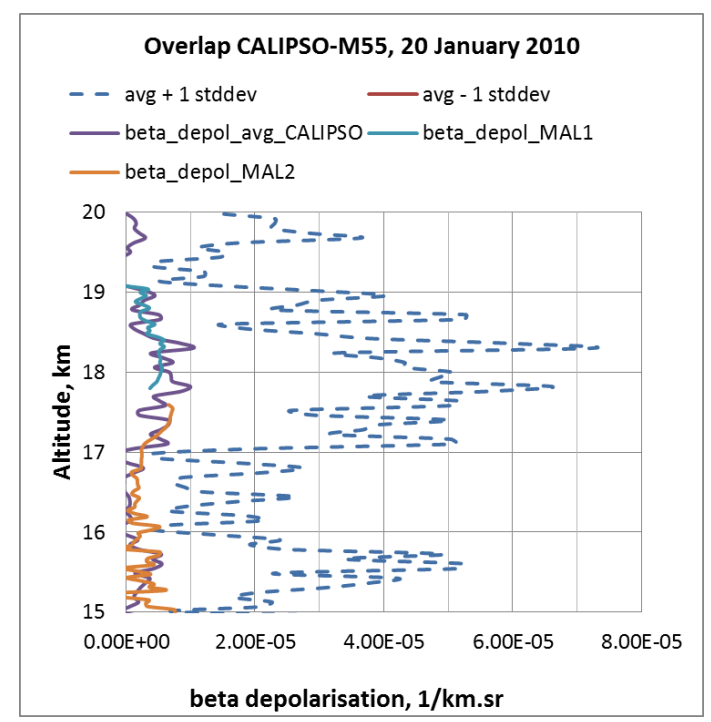

Figure S7: Comparison at PSC altitudes: Profiles of the cross-polarized component of the backscatter coefficient as derived from the measurements of the CALIPSO lidar, MAL1 and MAL2. Averaging period from $35800 \mathrm{~s}$ to 36600 s UTC of the flight from 20 January 2010.

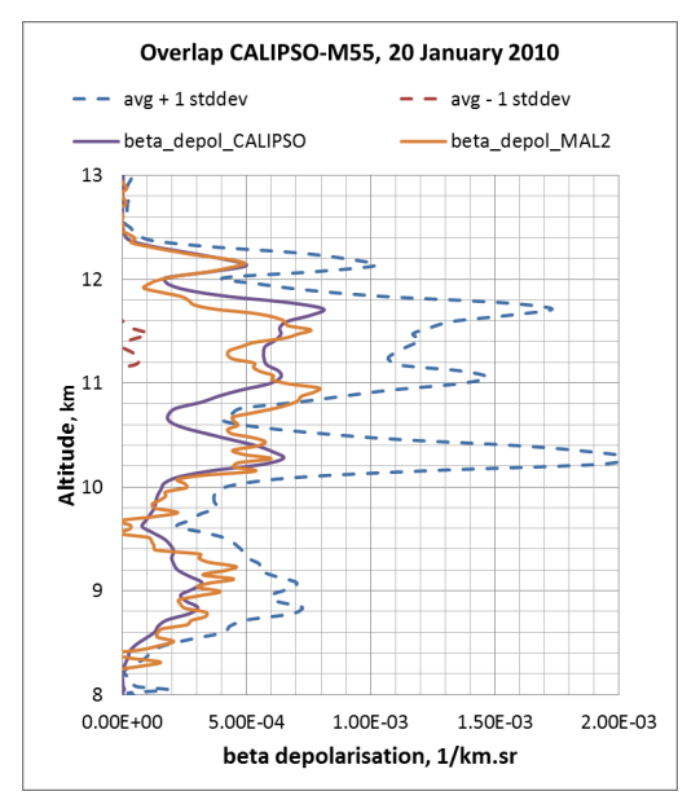

Figure S8: Comparison at cirrus cloud altitudes: Profiles of the cross-polarized component of the backscatter coefficients obtained from the measurements of the CALIPSO lidar, MAL1 and MAL2 on the flight from 20 January 2010. (Averaged from 35800 s to 36600 s UTC.)

\section{References:}

Achtert, P., and Tesche, M.: Assessing lidar-based classification schemes for polar stratospheric clouds based on 16 years of measurements at Esrange, Sweden, J. Geophys. Res. Atmos., 119, 13861405, doi:10.1002/2013JD020355, 2014. 
Behrendt, A., Nakamura, T.: Calculation of the calibration constant of polarization lidar and its dependency on atmospheric temperature, Opt. Express, 10, 805-817, http://dx.doi.org/10.1364/OE.10.000805, 2002.

Borrmann, S., Thomas, A., Rudakov, V., Yushkov, V., Lepuchov, B., Deshler, T., Vinnichenko, N., Khattatov, V., Stefanutti, L.: Stratospheric aerosol measurements in the Arctic winter of 1996/1997 with the M-55 Geophysika high-altitude research aircraft, Tellus, 52B, 1088-1103, 2000.

Borrmann, S., Kunkel, D., Weigel, R., Minikin, A., Deshler, T., Wilson, J. C., Curtius, J., Volk, C. M., Homan, C. D., Ulanovsky, A., Ravegnani, F., Viciani, S., Shur, G. N., Belyaev, G. V., Law, K. S., Cairo, F.: Aerosols in the tropical and subtropical UT/LS: In-situ measurements of ultrafine particle abundance and volatility, Atmos. Chem. Phys. 10, 5573-5592, 2010.

Cairo, F., Donfrancesco, G. D., Snels, M., Fierli, F., Viterbini, M., Borrmann, S., Frey, W.: A comparison of light backscattering and particle size distribution measurements in tropical cirrus clouds. Atmospheric Measurement Techniques, 4(3), 557-570, 2011.

Cairo, F., Di Donfrancesco, G., Adriani, A., Pulvirenti, L., Fierli, F., Comparison of various linear depolarization parameters measured by lidar. Applied Optics, 38(21), 4425-4432., 1999.

Collis, R. T. H., Russell P. B.: Lidar measurement of particles and gases by elastic backscattering and differential absorption, Laser monitoring of the atmosphere, 71-151, Springer Berlin Heidelberg, 1976.

Corte, V. D., Rietmeijer, F., Rotundi, A., Ferrari, M., and Palumbo, P.: Meteoric $\mathrm{CaO}$ and carbon smoke particles collected in the upper stratosphere from an unanticipated source, Tellus B, 65, http://www.tellusb.net/index.php/tellusb/article/view/20174, 2013.

Ebert, M., Weigel, R., Grooß, J.-U., Kandler, K., Molleker, S., Günther, G., Vogel, B.,Weinbruch, S., and Borrmann, S.: Characterization of refractory stratospheric aerosol particles collected within the polar vortex and polar stratospheric clouds - RECONCILE / ESSENCE, in preparation, Atmospheric Chemistry and Physics Discussion, 2015.

Freudenthaler, V., Esselborn, M., Wiegner, M., Heese, B., Tesche, M., Ansmann, A., Muller, D., Althausen, D., Wirth, M., Fix, A., Ehret, G., Knippertz, P., Toledano, C., Gasteiger, J., Garhammer, M. Seefeldner, M.: Depolarization ratio profiling at several wavelengths in pure Saharan dust during SAMUM 2006, Tellus B, 61: 165-179. doi: 10.1111/j.1600-0889.2008.00396.x, 2009.

Matthey, R., Mitev, V., Mileti, G., Makarov, V. S., Turin, A. V., Morandi, M., Santacesaria, V.: Miniature aerosol lidar for automated airborne application. 44-53, In AeroSense 2000, International Society for Optics and Photonics, 2000.

Mitev, V., Matthey, R., Makarov, V.: Miniature backscatter lidar for cloud and aerosol observation from high altitude aircraft, Rec. Res. Devel. Geophys, 4, 207, 2002.

Mitev, V., Poole, L., Pitts. M., Matthey, R.: Comparison case between CALIPSO lidar and MAS on the M-55 Geophysica during the RECONCILE campaign, $26^{\text {th }}$ International Laser Radar Conference, 25-29 July 2012, Porto Heli, Greece, 2012. 
Pitts, M. C., Poole, L. R., Thomason, L. W.: CALIPSO polar stratospheric cloud observations: secondgeneration detection algorithm and composition discrimination, Atmos. Chem. Phys., 9, 7577-7589, 2009.

Snels, M., Cairo, F., Colao, F., Di Donfrancesco, G.: Calibration method for depolarization lidar measurements, International Journal of Remote Sensing, 30(21), 5725-5736, 2009.

Young, A.: "Revised depolarization corrections for atmospheric extinction," Appl. Opt. 19, 3427-3428 (1980).

Warren, J., Achilles, C., Todd, N., Bastien, R., and Zolensky, M.: Particles from Collectors L2071, L2076, L2079, L2083 and W7068, Cosmic Dust Catalog, 18, http://curator.jsc.nasa.gov/dust/cdcat18. 